\title{
Tailoring Technology-Enhanced Learning Arrangements to Individual Needs
}

\author{
doi:10.3991/ijac.v2i2.833 \\ L. Thomas ${ }^{1}$, S. Trapp ${ }^{1}$, and S. Möbs ${ }^{2}$ \\ ${ }^{1}$ Fraunhofer Institute for Experimental Software Engineering, Kaiserslautern, Germany \\ 2 Dublin City University, Dublin, Ireland
}

\begin{abstract}
Efficiency and sustainability of technology-enhanced learning is increasingly guaranteed by target-group and subject-specific arrangements of learning-conducive elements. Self-learning phases are combined with synchronous or asynchronous online collaboration and cooperation, as well as with face-to-face training sessions. In the "Up2UML" project funded by the Leonardo-da-Vinci initiative, partners from Ireland, Romania, Bulgaria, France and Germany developed an approach for designing and tailoring learning arrangements for the software modelling domain. This paper describes how such a tailoring can be set up, putting a special focus on the design of learning scenarios (IMS Learning Design) in conjunction with the design of learning media (eduDocBook).
\end{abstract}

Index Terms-Blended learning, reuse, reconfiguration, customization.

\section{Motivation}

Traditional e-learning requires the capability to find one's own way in self-directed learning processes and it demands a high level of self-motivation. Studies on acceptance and usage of e-learning have shown self-learning competences and self-motivation as being the biggest challenge [1]. In order to evade loss of motivation and to enhance sustainability of learning, training sessions need to be carefully organized and include a well defined mix of activities [2]. In this respect, there is not much difference between traditional learning in a classroom (face-toface communication) and with support from printed textbooks, and learning with online materials (e.g. SCORM packages) and computer-mediated communication (e.g. Adobe Acrobat Connect, E-Mail). By including various teaching methods and activity types other than mere selfpaced learning, e-learning was turned into a more successful method called blended learning.

For those who are experienced in building blended learning arrangements, it still remains difficult to create a reasonable mix of learning elements for each target group, especially if the learning material - which is still an important part within such scenarios, is presented in a rather static way. In most cases, the trainer cannot just cut out bits and pieces, add new material and combine everything in order to create a new course or adapt the contents to the participants' special needs. This would require highly flexible and modularized material, which is hard to develop with the current approaches. A study from the ELearning Guild [3] showed that namely repurposing of content is one of the features that are urgently demanded; nevertheless it is one of the least supported by common commercial authoring tools (e.g. Adobe Flash, MS Word etc.).

In this article, an approach for tailoring technology enhanced learning arrangements to the needs of specific target groups and the requirements of each subject is presented. The article puts a special focus on the eduDocBook format for content development, which is based on the worldwide accepted standard in publishing DocBook. It shows how eduDocBook can be used to tailor learning material to the individual needs of each trainer and training session and how this is supported by easy to handle tools.

\section{RECONFIGURATION AND REUSE AS A CHALlENGE FOR CONTENT DEVELOPMENT}

The production of digital resources and especially learning content is often connected with high costs and expenditure of time: designers and authors team up to develop a general concept and create templates for implementation; agencies or internal service departments implement those concepts. This is why new online learning content is usually produced for large user groups that can be addressed through efficient communication channels. In the last few years, human resources management used to be interested in technology-enhanced learning mostly for lowering their costs per student. In order to achieve substantial savings, the same content has to be offered to a large number of students. This kind of profit perspective, however, disregards the content lifecycle, which includes, for example, the reuse of content in various media formats, or the easy update and repurposing of existing content whenever new requirements appear. At a time where e-learning is established as an important component of the media mix for training and helpdesk purposes, where companies and education institutions process a lot of existing content and continuously produce new content, the development and exploitation of reusability potentials becomes one of the key business factors.

Reuse and reconfiguration of content is still a central topic for further development of learning media production. Thomas and Ras [4] outlined in their analysis that most of the applied techniques for the development of learning media neglect the aspect of reuse and repurposing. In his dissertation [5], Hoermann draws the conclusion that common authoring tools often impede reuse through monolithic data formats for the storage of learning objects.

The challenges for reuse and repurposing of content are centered around three main areas: application context, media format, and organization. 


\section{ApPLICATION CONTEXT}

Learning content is produced to reach certain goals, for example "We want our employees to make fewer mistakes when entering data". Throughout the concept phase and the technology selection the content is often shaped to suit a certain usage scenario, e.g. for being used in self-paced learning. If the context changes, e.g. from usage in selfpaced learning by car mechanics to usage at a helpdesk for customers by a supplier to the automotive industry, the media have to be reworked at great expense. Consequently a special challenge in reuse is to develop content in a way that allows as many different reuse scenarios as possible.

Such repurpose of content throughout different applications requires a very thorough analysis and planning of possible application scenarios. Only with content that is created according to these principles right from the beginning, application across settings and formats becomes feasible. If for example material for self-paced learning constantly refers to "the course", it is strictly limited to this single application scenario.

\section{MEDIA FoRMAT}

Reusability across media formats is a further challenge. Content which is produced for a specific media format should also be applicable in different formats: an online course for instance should also be printable or displayable in a completely different format on a mobile device.

A central aspect for reuse across media formats is the single-source multimedia publishing approach. This concept requires all data and documents to be stored as redundant-free as possible in a central place. The consistent separation of the three text dimensions, content, structure and layout is essential in this respect, because only then a reuse across media formats is possible. Corresponding storage and subsequent transformation of the data will enable any output format.

From the area of electronic support systems, the socalled electronic performance support system (EPSS) approach [6] has evolved, describing all technical systems and media that support the user while working with a(n) (electronical) system: EPSS is "an integrated electronic environment that is [...] structured to provide immediate, individualized on-line access to the full range of information, software, guidance, advice and assistance, data, images, tools, and assessment and monitoring systems to permit job performance with minimal support and intervention by others." [6]. In the area of software products this means for example that an EPSS usually consists of a collection of documents (e.g. handbooks), help systems (e.g. online help), training materials (e.g. WBT) as well as further support. The media contribute to enabling the user to do the working tasks more efficiently. Learning content in this context is considered as a component supporting the user in addition to printed and online media.

Abstracting from these different media formats in the EPSS approach and looking at the complete scenario from the media format spanning perspective of single-source publishing, one can notice that the documents considered in the EPSS approach contain a variety of information which can be used in multiple media formats and in a reuse context (cf. [7]). The main goal of all the components of the EPSS is to support users performing their tasks. This requires that all components are optimized towards their media-specific advantages and the needs of the target group. Nevertheless there are many common aspects suitable for reuse. Quality requirements exist in a similar way: for learning systems verbal expression and comprehensibility, a clear structure, supporting media, easy navigation and quick access to information etc. as well as help system and online or offline documentation are similarly important. Furthermore, especially in SMEs nearly all parts of an EPSS are written by the same author and hence should provide a consistent usage of verbal styles. On the other hand there are also differences besides the similarities: while learning media are in general designed to provide a stream of information which the learners work through, from start to finish, e.g. to pass the final exam, one can assume in general a singular use of several parts for an online help or handbook. Online help is probably read only by few users from beginning to end; a user would expect an explicit list of learning goals in a courseware, but this would not make much sense in an online help, because here an encyclopedic access is prevalent. When developing an EPSS, a maximized reusability as well as aspects regarding specific requirements on media format, content and structure have to be considered accordingly.

\section{ORganization}

Organizational issues play an essential role for the success of reusability. Ultimately it is not sufficient that reuse is possible on the technical and content level. It has to be clarified on which level and organizational unit reusability should be carried out and under which conditions. The coverage could be delineated as follows:

- Project internal reuse: This is the easiest form of reuse, which basically targets the solution of technical and content-related problems, the coordination of authors (editorial guide) as well as intelligent concepts, storage and development of content.

- Organization-wide reuse: As soon as reuse leaves the area of the project, the lecturer, the faculty or the working group, all external legal jurisdiction, the allocation and consideration of rights of users become very important. Enterprises trying to establish organization-wide accessibility and use of content often face the problem that experts are reluctant to provide content, as they feel that by giving away their expertise their value will decrease.

- Cross-organizational reuse: Similar to the organization-wide reuse, the rights of users play again a major role. Even more than in other dimensions of reuse, the cross-organizational reuse requires agreements by contract, and an exchange of resources if applicable. Platforms such as educanext (www.educanext.org) which aim at a cross-organizational exchange of learning materials therefore operate with a vast license and usage rights management which enables the contributor to control the conditions of use by others.

This short analysis shows that reuse is a central challenge for the content lifecycle and that the problem can take on a number of dimensions. The main attention in this paper is on technical questions as outlined in the chapters on application context and media format. These will be discussed under special consideration of project internal reuse and a first method of resolution is presented. The necessity of a systematic development process and the intelligent coordination of authors has been pointed out 

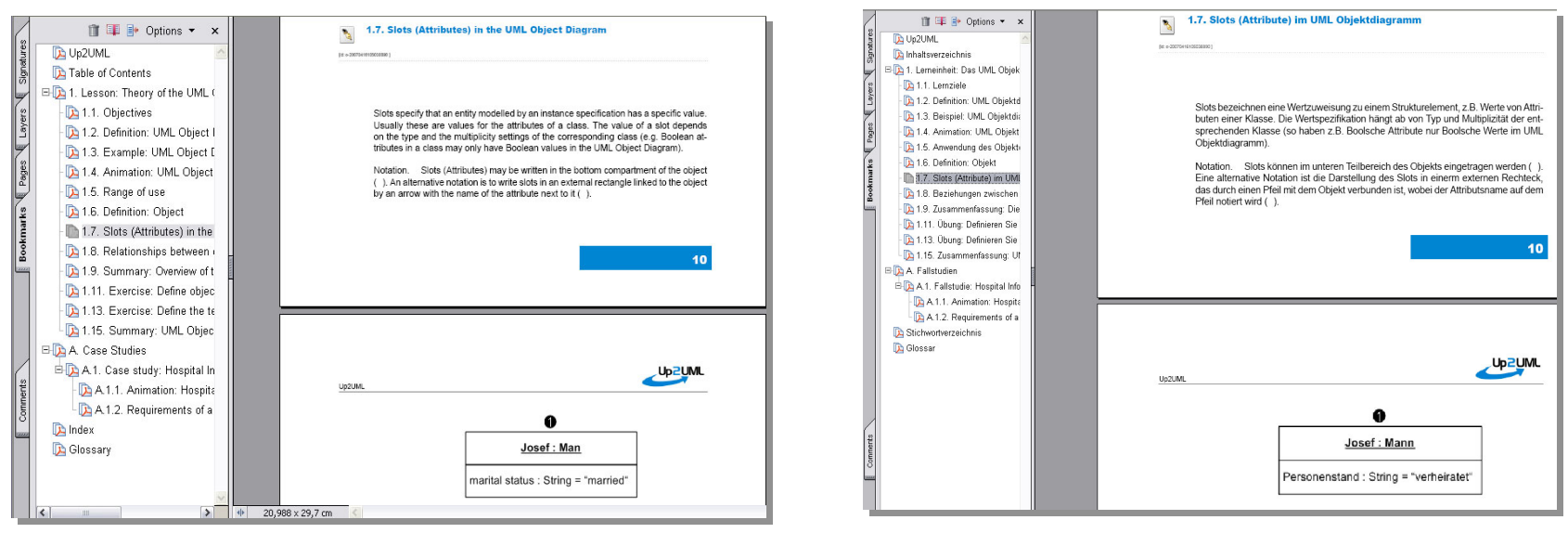

Figure 1. eduDocBook in multilingual PDF presentation

elsewhere [8] and will not be further explored here. In the following chapter, the idea of the eduDocBook as an exchange format is presented. Then the modular content production with this format is discussed and experiences and developments from the current project are presented. Finally the possibilities and limitations of the approach are discussed and an outlook on future work is given.

\section{USAGE OF WORLDWIDE STANDARDS FOR THE DESIGN OF MOdUlar LEARNing MEDIA. THE (EDU)DOCBOOK APPROACH}

DocBook [9] is an XML-dialect, developed as an exchange format for (software) documentation in the early 1990s and has reached worldwide distribution. It contains more than 400 general and more software-specific elements. An active community not only provides support for DocBook but also a number of tools for the creation of files as well as the transformation in various output formats. Due to its worldwide distribution, DocBook presents a successful example for the development of structured formats for documents for developers of XML. Thus developers of the educational modeling language (EML) report that DocBook was the model for developing their XML-dialects [10]. A wider dissemination of DocBook in the area of learning media production has not been reported so far.

According to Google, the term "eduDocBook" was first mentioned in a posting on a CETIS mailing list in June 2003. The discussion between Wilbert Kraan and Pierre Gorissen focused on whether and how SCORM should deal with the modeling of the actual learning content and if something like a universal content markup language was required. Furthermore, the discussion looked at the elements of DocBook and the idea of content denotation, originally contained in the EML of the Open University of the Netherlands, but which had been abandoned on the way to standardization (IMS LD) due to its complexity.

eduDocBook as developed and used at Fraunhofer IESE is an extension and specialization of DocBook, which recommends the language especially for the education sector. The previous and future development of eduDocBook follows primarily the following main ideas:

- If possible only minimal adjustments to DocBook should be made, so to ensure that the contents stay compatible with the standard. If necessary, extensions are included in a way that enables filtering them with transformation provided, to receive a DocBook in "pure" format.

- All options for specialization of elements (e.g. using the role attribute) should be checked before extensions to the DocBook are added. Domain-specific extensions should be avoided if possible. (e.g. <para role="definition" $>$ and not < definition $>$ ).

- Through intensive adaptation of transformations, learning media specific output formats, like, e.g. WBT format, are produced. In the process low-key standard technologies, freely available and adjustable tools are used.

In effect the currently available version of the eduDocBook mainly allows fine-granular modeling of sequential-hierarchical learning content (book, chapter, section, reference etc). The pages on information modeling in particular provide the following options:

- Markup of target group specific content (e.g. userlevel="novice") and content varieties (e.g. condition="course").

- Assistance of relations within documents (e.g. <eduRelationtype="isExerciseTo"

linkend="nodeid"/>).

- Modeling of exercises on element specialization (e.g. <itemizedlist role="mc-exercise">), automatic implementation of single forms of exercises of noninteractive (printing) formats.

- Heavily modularized storage and reuse of content.

- Integration of meta data (among others LOM) for the search of content components.

- Building-tool based on open source software .

- All other additional options provided by DocBook: glossary, index, directories, function references, bibliographies.

Successful implementations in industry and higher education prove that the eduDocBook format in combination with the accompanying tools enables the detailed markup of a number of formats and provides output in multiple configurations and media formats [8][11].

\section{CONTENT PRoduction WiTh (EDU)DocBooK}

On a technical level, the (edu)DocBook-based singlesource publishing approach differentiates four consecutive layers which are passed successively for compilation and processing of documentation [8]: 


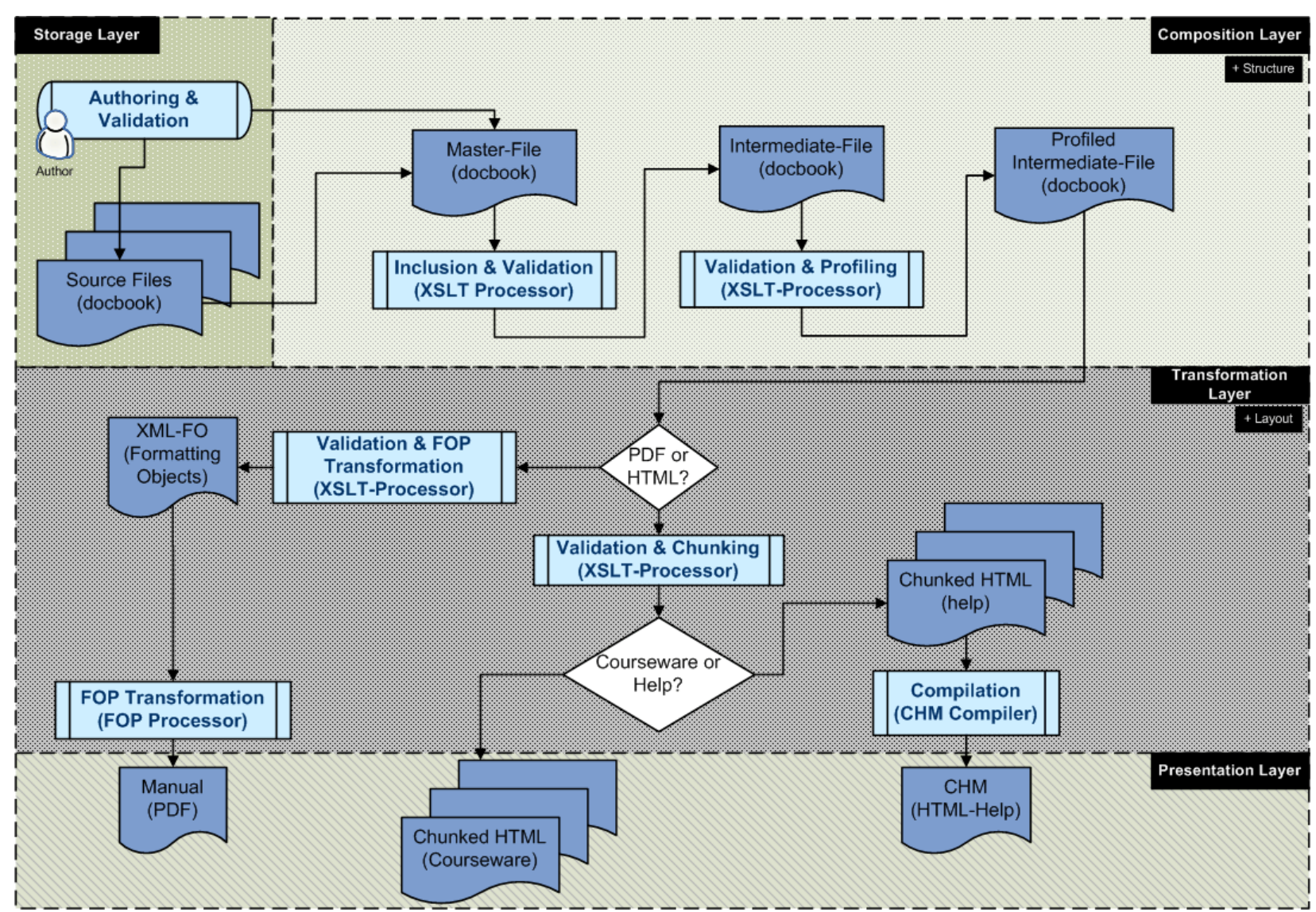

Figure 2. DocBook production process [7]

1) Storage layer: On this layer the components of the content are created in XML and retained for further processing.

2) Composition layer: This layer provides the aggregation of the components into a completed, valid DocBook document, which is used as a foundation for further processing.

3) Transformation layer: On this level the aggregated documentation is transformed into the desired output format.

4) Presentation layer: This is the layer that presents the formatted documentation to the user.

On the storage layer, which is responsible for creation and allocation of documentation, components authors will typically work with authoring tools such as an XML-editor or an IDE tool such as Eclipse. The components are for example retained in a CSV/SVN repository for further processing. Using technologies such as xinclude, xpointer, xml catalogue, conref etc. enables an extensive modularization of information as well as the reuse on a technical level. An XSLT processor is the central tool on the composition and transformation layer. It transforms the valid XML file of documentation, the profiled intermediate file, rule-based to HTML or XSL-FO. There is free software available for this such as XSLTproc, Xalan and Saxon. Apache FOP can be used to transform XSL-FO files into PDF files - although it only provides restricted possibilities. Professional results can be achieved using commercial products such as RenderX XEP or Antennahouse XSL Formatter. For presentation and allocation of the final documentation and media, a content management system
(CMS) or, in case of educational software, a learning management system (LMS) can be used. Over a transformation IMS manifesto files can be written from DocBook, which support the import into an LMS using the SCORM interface. The complete production process is largely automated and is controlled by a transformation assistant. The application initially queries the necessary data from the user and subsequently starts the transformation process for the wanted data and media formats.

Within the project "Upskilling to Object-Oriented development with the UML" (Up2UML), this form of content production was followed and further improved. In addition a specific WBT output format for eduDocBook with a more attractive and functional interface for the presentation of DocBook content was developed. This allows learners quick and easy access to the content. The SCORM export facilitates import into any of the current LMS such as Moodle.

\section{VIII.THE COURSECOMPOSER APPLICATION FOR THE PROdUCTION OF RECONFIGURABLE LEARNING MEDIA}

The developed content modules on the UML2 were stored as largely independent modular information objects and enriched with metadata. The overall goal was to enable future "reusers" of the components, such as trainers, publishing houses and course providers, to put together individual courses. A trainer may, for example, use the content developed in the project, for preparing a presence unit by using an online course on fundamentals of UML diagrams, and later on provide exercises together with a case study, handed out to the participants at the end of the workshop. The modular design within Up2UML supports 
the customization of existing blended learning concepts and the reconfiguration of courses and help, for example, focusing on specific types of UML diagrams.

The term reconfiguration of content in this paper refers in general to "authoring by aggregation" as described by [12]. Ultimately reconfiguration describes the combination of content from existing content components (aggregation) or the change of this combination as well as transfer to different dimensions of reuse (reconfiguration).

The creation process of learning media in Up2UML occurs in two steps: initially, content in modular form is created and stored. In a second step, these components, socalled content objects, are aggregated into didactically meaningful arrangements, including various access support tools (e.g., an index or advanced organizer). In the beginning, a local solution was used as an aggregation tool together with a tool for reconfiguration of content. During that stage the idea arose to apply the same processes to a web server and thus enable an even more comfortable combination and reconfiguration of educational media. This application, called Course Composer allows users to comfortably develop or reconfigure didactically motivated arrangements of content objects - factually these are XML files available in SVN in eduDocBook format. Subsequently, the combinations run through a number of transformations to be available for download in the desired output format, with the desired content (e.g. target group specific data).

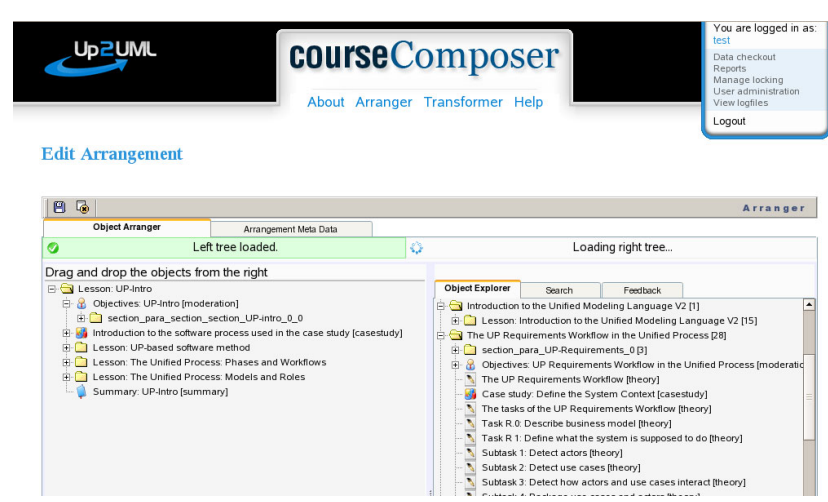

Figure 3. CourseComposer as part of "Up2UML"

Due to the use of innovative technologies, the CourseComposer provides an intuitive and easy to use interface. It is for example possible to put together courses by drag and drop using the so-called "Arranger" in CourseComposer. You can also move nodes, lectures, chapters etc. with the mouse. The so-called "Transformer" transfers the courses assembled with a few clicks into the desired output format. After completion of the transformation, the courses can be made available to the students in different formats, WBT (HTML) and PDF, as well as in different languages.

\section{Conclusions}

The analysis showed that tailoring educational content to the needs of each individual course is still an unsolved problem that, if solved, would provide an important stimulation of student motivation within blended learning arrangements. Furthermore, reuse and repurposing of contents has several potentials for the enhancement of elearning, since similar technologies could be used to deliver truly personalized learning experiences within adap- tive learning environments. eduDocBook as well as related tools provide solutions that enable trainers or course providers to tailor learning arrangements to their individual needs. In contrast to the many proprietary solutions (e.g. LMML, eEML, $<\mathrm{MI}>$ ), eduDocBook is built on basis of an established standard, worldwide in use and with a very active user group, as well as numerous related technologies. Due to the distribution and use of standard technologies, eduDocBook appears to be medium-term futureproof.

The described approach for creation and reconfiguration of content based on eduDocBook has proven valid within an industry application and a research project with varying goals. It is definitely also suitable for other technical or scientific topics, and should be further applied and developed. Although the use of DocBook in social sciences and humanities has been suggested sporadically [13], there are only a few experience reports about the suitability of the standard for the markup of corresponding texts, e.g. for medicine, law etc. [14]. As a result of flexible adjustment and specialization of DocBook - which increases further with the upcoming version 5 - it can be assumed that such solutions will become more widely spread.

Even if eduDocBook has proven to be an applicable markup language in the mentioned contexts, its low specialization in the area of educational media modeling is also an inherent problem of eduDocBook: its ability to describe exercises in a semantically accurate way is somehow limited. Complex didactical scenarios, often demanded, but rarely implemented in practice, can only be realized rudimentarily with the textbook logic implemented in eduDocBook. Furthermore, the feasibility of educational modeling with eduDocBook outside the described projects has to be examined. It is necessary to explore whether the techniques also work in the context of further blended learning projects and if the expected results can be achieved.

\section{REFERENCES}

[1] M. Keller and A. Back, "Blended-Learning-Projekte im Unternehmen". Arbeitsberichte des Learning Center der Universität St. Gallen, 3/2004.

[2] S. Möbs and S. Weibelzahl, "Towards a good mix in Blended Learning for Small and Medium-sized Enterprises - Outline of a Delphi Study", in E. Tomadaki and P. Scott (eds), Innovative Approaches for Learning and Knowledge Sharing, EC-TEL 2006 Workshops Proceedings, Crete, Greece. Milton Keynes, Open University, p. 10-17.

[3] The eLearningguild: "The Content Authoring Research Report 2005". http://www.elearningguild.com/pdf/1/Oct05-contentauthor. pdf (13.06.06).

[4] L. Thomas and E. Ras, "Wiederverwendungsorientiertes Content Authoring nach dem Single-Source Prinzip", in M. Mühlhäuser, G. Rößling, and R. Steinmetz (eds), DeLFI 2006, 4. e-Learning Fachtagung Informatik, GI-Edition, München (2006), p. 159-170.

[5] S. Hoermann, "Wiederverwendung von digitalen Lernobjekten in einem auf Aggregation basierenden Autorenprozess". Dissertation, Technische Universität Darmstadt, Darmstadt (2005).

[6] G. Gery, Electronic Performance Support System. Gery Association (1991).

[7] L. Thomas and E. Ras, "Courseware Development Using a SingleSource Approach", in Proceedings of the World Conference on Education Multimedia, Hypermedia and Telecommunications, EdMedia, Montreal (2005); p. 4502-4509.

[8] I. Grützner and L. Thomas, "Systematische Entwicklung von Medien zur Benutzerunterstützung und -schulung mit einem XML 
basierten Single-Source-Ansatz". Multikonferenz Wirtschafts-informatik, Dresden (2006).

[9] N. Walsh and L. Muellner, DocBook. O'Reilly, 1999.

[10] R. Koper and B. Olivier, "Representing the learning design of units of learning", in Educational Technology \& Society, 7 (3), p. 97-111.

[11] I. Grützner, L. Thomas, and S. Steinbach-Nordmann, "Building reconfigurable multilingual training media", in A. Méndez-Vilas, A. Solano Martin, and J. Mesa Gonzále (eds), Current Developments in Technology-Assisted Education (2006) - Vol. 3, p. 1944-1948.

[12] S. Hoermann, C. Rensing, and R. Steinmetz, "Wiederverwendung von Lernressourcen mittels Authoring by Aggregation im ResourceCenter", in J.M. Haake, U. Lucke, and D. Tavangarian (eds), DeLFI 2005: 3. Deutsche e-Learning Fachtagung Informatik, GIEdition, München (2005), p. 153-164.

[13] H. Bunke, "Schreibt strukturiert! XML und Docbook in Sozialund Geisteswissenschaften". http://hbxt.org/edutech/docbook-ingeisteswissenschaften (17.03.2007).

[14] A.K. Merz, F. Rockmann, C. Schwarz,and C.M. Reng, "Rechnergestützte Lehr- und Lernsysteme in der Medizin. "MedicCaseML: XML Austauschformat für CBT Systeme in der medizinischen Aus- und Weiterbildung", in J. Bernauer, M.R. Fischer, J. Leven, F. Puppe, and M. Weber, Rechnergestützte Lehr- und Lernsysteme in der Medizin, p. 67-76, Shaker Verlag: Berlin (2002).

\section{AUTHORS}

L. Thomas was with the Fraunhofer Institute for Experimental Software Engineering, Kaiserslautern, Germany. He is now with the e-learning department of Verein Deutscher Zementwerke e.V., Forschungsinstitut der Zementindustrie, Düsseldorf, Germany (e-mail: tl@vdz-online.de).

S. Trapp is with the Fraunhofer Institute for Experimental Software Engineering, Kaiserslautern, Germany. (e-mail: sonja.trapp@iese.fraunhofer.de).

S. Möbs is with the Performance Engineering Lab at the School of Electronic Engineering at Dublin City University, Dublin , Ireland (e-mail: sabine@eeng.dcu.ie).

This work was carried out in the framework of the project "Upskilling to Object-Oriented Development with the UML" (Up2UML) and was supported by the European program LEONARDO DA VINCI 2005 2007 (Project No. PP 146369).

Manuscript received 09 February 2009. Published as submitted by the authors. 